\title{
8th Grade Completion
}

National Cancer Institute

\section{Source}

National Cancer Institute. 8th Grade Completion. NCI Thesaurus. Code C67130.

Indicates that 8th grade is the highest level of educational achievement. 